\title{
Efeitos de Intervenção Comportamental Intensiva Realizada por Meio da Capacitação de Cuidadores de Crianças com Autismo
}

\author{
Camila Graciella Santos Gomes ${ }^{1,2, *}$ (D), Deisy das Graças de Souza, ${ }^{2,3}$, Analice Dutra Silveira ${ }^{4}$ (D), \\ Aline Chaves Rates ${ }^{4}$, Gabrielle Chequer de Castro Paiva ${ }^{1}, \&$ Natália Paiva de Castro ${ }^{1}$ \\ ${ }^{1}$ Faculdade de Ciências Médicas de Minas Gerais, Belo Horizonte, MG, Brasil \\ ${ }^{2}$ Instituto Nacional de Ciência e Tecnologia sobre Comportamento, Cognição e Ensino, São Carlos, SP, Brasil \\ ${ }^{3}$ Universidade Federal de São Carlos, São Carlos, SP, Brasil \\ ${ }^{4}$ CEI Desenvolvimento Humano, Belo Horizonte, $M G$, Brasil
}

\begin{abstract}
RESUMO - O estudo avaliou os efeitos da Intervenção Comportamental Intensiva, realizada por meio da capacitação de cuidadores, no desenvolvimento de crianças com autismo. A pesquisa foi realizada a partir dos dados de crianças atendidas por um centro brasileiro especializado. Os participantes do Grupo 1 eram 22 crianças que finalizaram o primeiro ano de intervenção intensiva. Onze crianças que não fizeram a intervenção intensiva compuseram o Grupo 2, para finns de análise de dados. Os resultados indicaram ganhos significativos em todas as áreas do desenvolvimento das crianças com autismo que passaram pelo primeiro ano de Intervenção Comportamental Intensiva, enquanto as crianças do grupo controle apresentaram ganhos menos expressivos. Crianças mais novas, que falavam e que apresentavam sintomas mais brandos de autismo obtiveram melhores resultados.
\end{abstract}

PALAVRAS-CHAVE: autismo, cuidadores, análise do comportamento aplicada, intervenção comportamental intensiva

\section{Effects of Intensive Behavioral Intervention through Training of Caregivers of Children with Autism}

\begin{abstract}
The study evaluated the effects of Intensive Behavioral Intervention, conducted through training of caregivers, on the development of children with autism. The analysis was based on data of children treated by a specialized Brazilian center. Participants in Group 1 were 22 children who completed the first year of intensive intervention. Eleven children, who did not receive intensive intervention, were assigned to Group 2, for data analysis purposes. The results indicated significant gains in all areas of the development of children with autism who have gone through the first year of Intensive Behavioral Intervention, while children in the control group showed less impressive gains. Younger children, who spoke and who had milder symptoms of autism, obtained better results.
\end{abstract}

KEYWORDS: autism, caregivers, applied behavior analysis, intensive behavioral intervention

O autismo é um transtorno do desenvolvimento infantil caracterizado por alterações nas interações sociais e na comunicação, bem como pela presença de interesses fixos, restritos e intensos, e comportamentos repetitivos (American Psychiatry Association [APA], 2013). Até o momento, não há cura para o transtorno (APA, 2013; Gargiulo, 2006), porém, Intervenções Comportamentais Intensivas têm demonstrado efeitos significativos no desenvolvimento de pessoas com esse diagnóstico desde a década de 1980 (Boyd \& Corley, 2001; Campbell, Schopler, Cueva, \& Hallin, 1996; Dawson et al., 2010; Lovaas, 1987; Smith, 1999; Warren et al., 2011). A literatura considera como Intervenção Comportamental Intensiva (ICI) os modelos de intervenção caraterizados por estimulação individualizada (um educador para uma criança

\footnotetext{
*E-mail: camila.gomes@ceidesenvolvimentohumano.com.br

Financiamento: As autoras CGSG e DGS receberam apoio de FAPESP: 2014/50909-8; CNPq: 465686/2014-1; CAPES: 88887.136407/2017-00.

- Submetido: 22/03/2016; Revisado: 18/04/2017; Aceito: 23/05/2017.
} 
com autismo), realizados por muitas horas semanais (de 15 a 40 horas), por pelo menos dois anos consecutivos, que abrangem várias áreas do desenvolvimento simultaneamente e que são fundamentados em princípios de Análise do Comportamento (Green, 1996).

A literatura indica que os educadores podem ser estudantes, profissionais e familiares. Para viabilizar a quantidade de horas de intervenção necessárias, tem sido comum a atuação combinada desses tipos de educadores (e.g., Anderson, Avery, DiPietro, Edwards, \& Christian, 1987: pais, psicólogos, fonoaudiólogos e professores de educação especial; Dawson et al., 2010: profissionais e pais; Eikeseth, Smith, Jahar, \& Eldevik, 2002: professores; Lovaas, 1987: profissionais e estagiários; Sheinkopf \& Sielgel, 1998: pais; Sherman, Barker, Lorimer, Swinson, \& Factor, 1988: pais e profissionais; Smith, Buch, \& Gamby, 2000: pais e estagiários). A respeito das horas de intervenção, há indicações entre 15 e 40 h semanais, apesar de a maioria dos estudos indicarem de 30 a $40 \mathrm{~h}$ semanais por pelo menos dois anos consecutivos (Dawson \& Osterling, 1997; Dawson et al., 2010; Smith et al., 2000; Lovaas, 1987). Sobre o perfil da intervenção, a literatura indica a obrigatoriedade de estimular simultaneamente várias áreas do desenvolvimento e não focar em uma área específica (Green, 1996; Warren et al., 2011). Agregado a tudo isso, a intervenção tem que ser firmemente fundamentada nos princípios da aprendizagem operante (Skinner, 1953) e caracterizada como Análise do Comportamento Aplicada (Applied Behavior Analysis [ABA], Baer, Wolf, \& Risley, 1987). Tipicamente, é feita uma análise da função dos comportamentos que são relevantes e das condições de ensino, em termos de arranjos ambientais e de variáveis motivacionais (consequências reforçadoras) para promover o desenvolvimento desses comportamentos, se ausentes; e seu fortalecimento, se eles ainda ocorrem de maneira incipiente ou fraca (Skinner, 1968). A intervenção pode ser realizada em uma instituição especializada, na escola ou na residência da criança com autismo (Eikeseth et al., 2002; Reed, Osborne, \& Corness, 2007; Valenti, Cerbo, Masedu, De Caris, \& Sorge, 2010). Outro aspecto importante é que esse tipo de intervenção apresenta melhores resultados quando é realizada precocemente (com início até os 4 anos de idade), quando programa condições para garantir a generalização das habilidades aprendidas (para outros ambientes, pessoas ou situações) e quando conta com a participação dos pais (Aiello, 2002; Boyd \& Corley, 2001; Green, 1996).

O primeiro estudo sobre ICI aplicada ao tratamento do autismo foi publicado na década de 1980 (Lovaas, 1987) e descreveu o trabalho desenvolvido no projeto intitulado Early Intervention Project (EIP). Três grupos de crianças com autismo, que tinham idades abaixo de 4 anos no início do estudo, foram avaliados. O grupo experimental, composto por 19 crianças, foi exposto à ICI, com um educador para cada criança, por 40 horas semanais ou mais, por dois ou mais anos consecutivos. $\mathrm{O}$ tratamento consistiu, basicamente, no ensino simultâneo de habilidades em diversas áreas (e.g., comunicação, interação social, imitação, autocuidados) visando melhorar o desenvolvimento das crianças. Um grupo controle, composto por 19 crianças, recebeu intervenção comportamental mínima, por $10 \mathrm{~h}$ semanais ou menos. Um segundo grupo controle era composto por 21 participantes que foram tratados em outros centros de atendimento, os quais não realizavam intervenção comportamental ou outro tipo de intervenção intensiva e tampouco tinham contato com o projeto. Os resultados indicaram que $47 \%$ das crianças expostas à ICI tiveram redução significativa dos sintomas de autismo, apresentando desenvolvimento próximo ao esperado para a idade cronológica (próximo ao de uma criança típica, sem autismo); $42 \%$ tiveram uma redução acentuada dos sintomas e $11 \%$ continuaram com sintomas graves de autismo. Ou seja, aproximadamente $90 \%$ das crianças do grupo experimental apresentaram melhora no desenvolvimento e metade das crianças apresentou desenvolvimento próximo ao típico. Em estudo subsequente, realizado quando as crianças tinham aproximadamente 12 anos, constatou-se a manutenção desses resultados (McEachin, Smith, \& Lovaas, 1993).

Já as crianças do grupo controle que receberam intervenção comportamental mínima obtiveram resultados muito diferentes: $2 \%$ apresentaram desenvolvimento próximo ao típico, $45 \%$ tiveram uma redução dos sintomas e $53 \%$ continuaram com sintomas graves de autismo. As crianças do outro grupo controle, tratadas em outros centros de atendimento, também apresentaram resultados muito aquém dos obtidos pelas crianças do grupo experimental.

Os resultados descritos por Lovaas (1987) foram bastante criticados por pesquisadores que questionaram a validade do estudo em função de problemas na metodologia utilizada, especialmente em relação à distribuição dos participantes entre grupo controle e experimental, que não foi randômica, e falhas no controle de variáveis da intervenção em função da quantidade de horas semanais de terapia (Gresham \& MacMillan, 1998; Schopler, Short, \& Mesibov, 1989; Warren et al., 2011).

Porém, dezenas de estudos subsequentes replicaram parcialmente os resultados, indicando, no geral, melhora no desenvolvimento dos participantes após realizarem a ICI, inclusive quando comparados a outros tipos de terapias não intensivas ou não fundamentadas na Análise do Comportamento. Esses estudos descreveram resultados variados em relação à proporção dos ganhos da intervenção; mas, de modo geral, indicaram ganhos no desenvolvimento (Smith et al., 2000; Warren et al., 2011).

Por outro lado, a literatura também ressalta as dificuldades para a realização desse tipo de pesquisa: utilização de delineamento de grupo com distribuição randômica dos participantes, duração longa ( 2 anos ou mais), controle difícil de variáveis (muitas horas de intervenção e muitas pessoas envolvidas) e alto custo de execução (Boyd \& Corley, 2001; Smith, 1999; Warren et al., 2011; Weiss, 1999). 
Smith et al. (2000) afirmaram que apesar dos estudos sugerirem a necessidade de replicações com delineamentos experimentais mais rigorosos, as pesquisas existentes, combinadas com os diversos estudos de caso que descrevem os bons resultados da ICI (e.g., Maurice, 1993), indicaram a viabilidade desse tipo de intervenção e criaram uma demanda alta das famílias de pessoas com autismo por esse tipo de tratamento. Essa demanda encontrou dois problemas práticos: o elevado número de crianças com autismo (que é maior do que a quantidade de profissionais capacitados) e o alto custo financeiro desse tipo de intervenção (em função da quantidade necessária de horas semanais de terapia ou de ensino de repertórios adaptativos). Os autores ainda enfatizaram que se tornou inviável utilizar profissionais especializados para realizar todo o tratamento e que a solução mais comum para implementar a ICI tem sido empregar pais e estagiários para realizar a intervenção (terapeutas paraprofissionais), com orientação e supervisão de terapeutas comportamentais capacitados (Anderson et al., 1987; Dawson et al., 2010; McConachie \& Diggle, 2006,
Sheinkopf \& Sielgel, 1998; Sherman et al., 1988; Smith et al., 2000).

Diante do cenário atual de pesquisa e intervenção com essa população, o objetivo deste estudo é avaliar os efeitos da ICI realizada por cuidadores (pais, estagiários ou babás) especialmente capacitados para a tarefa sobre o desenvolvimento de crianças brasileiras com autismo. Para esta finalidade, a pesquisa utilizou delineamento de grupo e foi realizada a partir dos dados de crianças que foram atendidas em um centro brasileiro especializado no tratamento de pessoas com autismo. Foram analisados os dados do primeiro ano de ICI. A estratégia para medir os efeitos da intervenção consistiu em comparar o desempenho das crianças que receberam a ICI com o de outras crianças avaliadas (e reavaliadas) no mesmo centro, mas que, por razões diversas, não realizaram esse tipo de intervenção intensiva. A análise de dados produzidos por instituições especializadas foi empregada anteriormente por outros estudos da área (e.g., Harris \& Handleman, 2000; Luiselli, Cannon, Ellis, \& Sisson, 2000; Weiss, 1999).

\section{MÉTODO}

\section{Participantes}

Participaram do estudo 32 crianças que buscaram atendimento no Centro de Estudos e Intervenção para o Desenvolvimento Humano (CEI) entre 2011 e 2015. Todas as crianças tinham o diagnóstico de autismo, sem o diagnóstico de comorbidades, realizado por psiquiatras e neurologistas infantis especializados, independentes do CEI, seguindo critérios da CID-10 (Organização Mundial de Saúde, 1993), do DSM-IV-TR (APA, 2002), ou do DSM-V (APA, 2013). O critério escolhido dependia da preferência do médico que realizou o diagnóstico, sem qualquer interferência do CEI.

As crianças foram divididas em dois grupos para a análise dos resultados: o Grupo 1 (Tabela 1) era composto por 22 crianças que finalizaram o primeiro ano de ICI; o Grupo 2 (Tabela 2) era composto por 11 crianças que não fizeram a ICI porque as famílias desistiram do atendimento (P24, P28, P30) ou não aderiram ao tratamento (não seguiram as orientações dos terapeutas e não fizeram os registros das atividades: P26, P27, P29, P32). Outros participantes desse grupo permaneceram em atendimento de psicologia, realizado em consultório, uma hora por semana, caracterizado como terapia não intensiva (P23, P25, P31, $\mathrm{P} 21)$. Um dos participantes (P21) permaneceu em terapia não intensiva por 9 meses e posteriormente realizou a intervenção intensiva; por isso, seus dados foram inseridos nos Grupos 1 e 2. Todas as crianças frequentavam escolas comuns, cinco vezes por semana, totalizando 20 horas semanais, exceto P17, que não frequentava escolas.

Os cuidadores das crianças do Grupo 1 também participaram do estudo (um cuidador para cada criança) e eram estagiários de psicologia (P1, P2, P7, P17, P21, P22) ou pedagogia (P3, P13), babás (P4, P10, P11), mães (P5, P6, P8, P9, P12, P14, P15, P16, P18, P19) ou pai (P20). Do total de cuidadores, $13,6 \%$ eram babás; $36,4 \%$, estagiários e $50 \%$, pais. $13,6 \%$ concluíram até o ensino fundamental; $54,6 \%$, o ensino médio e $31,8 \%$, o ensino superior. Cuidadores das crianças do Grupo 2 não participaram do estudo porque não realizaram intervenção intensiva com as crianças desse grupo.

\section{Instrumentos de Avaliação}

Foram utilizados o Inventário Portage Operacionalizado (IPO; Williams \& Aiello, 2001), o Perfil Psicoeducacional Revisado (PEP-R; do inglês Psychoeducational ProfileRevised; Schopler, Reichler, Bashford, Lansing, \& Marcus, 1990) e a Childhood Autism Rating Scale (CARS; Schopler, Reichler, \& Renner, 1988). A pontuação (resultado) do IPO e do PEP-R permite comparar o desempenho da criança com autismo com o de uma criança típica, em termos de anos e meses.

O IPO, adaptado e operacionalizado para a população brasileira por Williams e Aiello (2001), com base no Portage Guide to Early Educaction Manual (Bluma, Shearer, Frohman, \& Hilliard, 1976) e em outros instrumentos do Portage Project, avalia o padrão de desenvolvimento infantil em cinco áreas: linguagem, socialização, desenvolvimento motor, cognição e autocuidados, em períodos de idade que vão de 0 a 6 anos. Esse instrumento avalia o desenvolvimento de qualquer criança, independentemente de algum tipo de diagnóstico. 
Tabela 1

Características Gerais dos Participantes do Grupo 1- Intervenção Intensiva: Participantes; Gênero; Idade e Presença de Fala Pré e Pós Intervenção; Pontuação na CARS, no PEP-R e no IPO Pré e Pós Intervenção; Número de Programas de Ensino; Horas de Intervenção Semanal; e Horas Semanais de Outras Terapias Não Intensivas.

\begin{tabular}{|c|c|c|c|c|c|c|c|c|c|c|c|c|c|c|}
\hline \multirow{2}{*}{$\mathbf{P}$} & \multirow{2}{*}{ Gênero } & \multicolumn{2}{|c|}{ Idade } & \multicolumn{2}{|c|}{ Fala } & \multicolumn{2}{|c|}{ CARS } & \multicolumn{2}{|c|}{ PEP-R } & \multicolumn{2}{|c|}{ IPO } & \multirow{2}{*}{ Programas } & \multirow{2}{*}{ Horas } & \multirow{2}{*}{ Outras } \\
\hline & & Pré & Pós & Pré & Pós & Pré & Pós & Pré & Pós & Pré & Pós & & & \\
\hline 1 & M & 1a $3 \mathrm{~m}$ & 1a $11 \mathrm{~m}$ & Não & Sim & - & - & 25 & 90 & 122 & 285 & 15 & 10 & 7 \\
\hline 2 & $\mathrm{M}$ & $2 \mathrm{a}$ & $2 \mathrm{a} 11 \mathrm{~m}$ & Não & Não & - & - & 10 & 37 & 97 & 159 & 17 & 20 & 1 \\
\hline 3 & M & $2 a$ & $3 a$ & Não & Sim & - & - & 18 & 57 & 123 & 213 & 22 & 10 & 1 \\
\hline 4 & $\mathrm{M}$ & $2 \mathrm{a} 2 \mathrm{~m}$ & $3 \mathrm{a} 3 \mathrm{~m}$ & Não & Não & - & - & 17 & 50 & 109 & 191 & 15 & 15 & 0 \\
\hline 5 & $\mathrm{~F}$ & $2 \mathrm{a} 10 \mathrm{~m}$ & $3 \mathrm{a} 9 \mathrm{~m}$ & Não & Sim & - & - & 24 & 46 & 115 & 201 & 15 & 15 & 1 \\
\hline 6 & M & $3 \mathrm{a} 1 \mathrm{~m}$ & $3 \mathrm{a} 11 \mathrm{~m}$ & Não & Sim & 37,5 & 28,5 & 37 & 82 & 165 & 262 & 7 & 10 & 4 \\
\hline 7 & M & $3 a 1 m$ & $4 \mathrm{a} 1 \mathrm{~m}$ & Sim & Sim & 31 & 27,5 & 35 & 88 & 205 & 299 & 20 & 15 & 2 \\
\hline 8 & M & $3 \mathrm{a} 5 \mathrm{~m}$ & 4a $5 \mathrm{~m}$ & Não & Sim & 26,5 & 27,5 & 48 & 87 & 191 & 320 & 29 & 15 & 2 \\
\hline 9 & M & $3 \mathrm{a} 6 \mathrm{~m}$ & 4a $9 \mathrm{~m}$ & Não & Não & 37 & 34,5 & 31 & 61 & 154 & 255 & 9 & 10 & 0 \\
\hline 10 & M & $3 \mathrm{a} 11 \mathrm{~m}$ & $4 \mathrm{a} 10 \mathrm{~m}$ & Não & Não & 32,5 & 30 & 32 & 50 & 166 & 224 & 18 & 15 & 1 \\
\hline 11 & M & 4a $11 \mathrm{~m}$ & $6 a$ & Sim & Sim & 31,5 & 31,5 & 66 & 83 & 244 & 299 & 10 & 10 & 3 \\
\hline 12 & $\mathrm{M}$ & 5a $2 \mathrm{~m}$ & 6a $1 \mathrm{~m}$ & Não & Não & 52 & 43,5 & 15 & 28 & 139 & 152 & 12 & 15 & 1 \\
\hline 13 & M & $5 \mathrm{a} 3 \mathrm{~m}$ & $6 \mathrm{a} 2 \mathrm{~m}$ & Não & Sim & 37,5 & 31,5 & 63 & 95 & 209 & 354 & 36 & 20 & 1 \\
\hline 14 & $\mathrm{~F}$ & $5 \mathrm{a} 7 \mathrm{~m}$ & $6 \mathrm{a} 7 \mathrm{~m}$ & Sim & Sim & 32 & 34,5 & 57 & 83 & - & - & 23 & 15 & 3 \\
\hline 15 & M & $5 \mathrm{a} 11 \mathrm{~m}$ & 6a $11 \mathrm{~m}$ & Sim & Sim & 37,5 & 38 & 40 & 95 & - & - & 16 & 15 & 0 \\
\hline 16 & $\mathrm{M}$ & $6 a$ & $6 \mathrm{a} 11 \mathrm{~m}$ & Não & Não & 43,5 & 34 & 41 & 63 & - & - & 13 & 10 & 3 \\
\hline 17 & $\mathrm{M}$ & $6 \mathrm{a} 3 \mathrm{~m}$ & $7 \mathrm{a} 3 \mathrm{~m}$ & Não & Não & 46,5 & 42,5 & 19 & 46 & - & - & 43 & 40 & 0 \\
\hline 18 & $\mathrm{M}$ & $6 \mathrm{a} 3 \mathrm{~m}$ & $7 \mathrm{a} 6 \mathrm{~m}$ & Não & Não & 47,5 & 43 & 27 & 52 & - & - & 18 & 15 & 1 \\
\hline 19 & M & $6 \mathrm{a} 11 \mathrm{~m}$ & $7 \mathrm{a} 11 \mathrm{~m}$ & Não & Não & 58 & 48 & 13 & 25 & - & - & 9 & 15 & 0 \\
\hline 20 & $\mathrm{M}$ & 7a 9m & $8 \mathrm{a} 9 \mathrm{~m}$ & Sim & Sim & 47 & 38 & 41 & 62 & - & - & 12 & 10 & 0 \\
\hline 21 & M & $8 \mathrm{a} 11 \mathrm{~m}$ & $10 \mathrm{a}$ & Sim & Sim & 36 & 27 & 85 & 104 & - & - & 15 & 15 & 2 \\
\hline 22 & $\mathrm{M}$ & $11 \mathrm{a} 4 \mathrm{~m}$ & $12 \mathrm{a} 3 \mathrm{~m}$ & Não & Não & 54,5 & 49,5 & 24 & 36 & - & - & 13 & 20 & 0 \\
\hline Médias & & 4a $8 \mathrm{~m}$ & 5a $8 \mathrm{~m}$ & $27,2 \%$ & $54,5 \%$ & 40,5 & 35,8 & 34,9 & 64,5 & 156,8 & 247,2 & 17,6 & 15,2 & 1,5 \\
\hline p-valor & & & & & & $0,002 *$ & $0,000 *$ & $0,001 *$ & & & & & & \\
\hline
\end{tabular}

Nota: $\mathrm{O}$ asterisco indica diferenças estatisticamente significativas $(\mathrm{p}<0,05)$, Teste de Wilcoxon

Tabela 2

Características Gerais dos Participantes do Grupo 2- Intervenção Não Intensiva: Participantes; Gênero; Idade, Presença de Fala, Pontuação na CARS e no PEP-R na Primeira (A1) e na Segunda (A2) Avaliação; Intervalo em Meses Entre as Avaliações A1 e A2; Horas Semanais de Outras Terapias Não Intensivas.

\begin{tabular}{|c|c|c|c|c|c|c|c|c|c|c|c|c|c|}
\hline \multirow{2}{*}{$\mathbf{P}$} & \multirow{2}{*}{ Gênero } & \multicolumn{2}{|c|}{ Idade } & \multicolumn{2}{|c|}{ Fala } & \multicolumn{2}{|c|}{ CARS } & \multicolumn{2}{|c|}{ PEP-R } & \multicolumn{2}{|c|}{ IPO } & \multirow{2}{*}{$\begin{array}{c}\text { Intervalo } \\
(\mathrm{A} 2-\mathrm{A} 1)\end{array}$} & \multirow{2}{*}{ Outras } \\
\hline & & $\mathrm{A} 1$ & $\mathrm{~A} 2$ & A1 & $\mathrm{A} 2$ & A1 & $\mathrm{A} 2$ & A1 & A2 & A1 & $\mathrm{A} 2$ & & \\
\hline 23 & M & $2 \mathrm{a} 5 \mathrm{~m}$ & $3 a 3 m$ & Não & Não & - & & 11 & 26 & 127 & 151 & 10 & 1 \\
\hline 24 & $\mathrm{~F}$ & $3 a 4 m$ & 5 anos & Não & Não & 45 & 10 & 6 & 14 & 101 & 167 & 20 & 0 \\
\hline 25 & $\mathrm{~F}$ & 3 a $5 \mathrm{~m}$ & $4 \mathrm{a} 3 \mathrm{~m}$ & Sim & Sim & 29 & 20 & 61 & 60 & 249 & 322 & 10 & 2 \\
\hline 26 & M & $3 \mathrm{a} 6 \mathrm{~m}$ & $4 \mathrm{a} 4 \mathrm{~m}$ & Não & Não & 38 & 10 & 42 & 40 & 193 & 194 & 10 & 1 \\
\hline 27 & M & $4 \mathrm{a} 9 \mathrm{~m}$ & $5 \mathrm{a} 9 \mathrm{~m}$ & Sim & Sim & 44 & 10 & 38 & 50 & 160 & 229 & 12 & 0 \\
\hline 28 & M & $5 \mathrm{a} 6 \mathrm{~m}$ & $7 \mathrm{a} 3 \mathrm{~m}$ & Não & Não & 30 & 12 & 58 & 41 & - & - & 21 & 1 \\
\hline 29 & M & $5 \mathrm{a} 10 \mathrm{~m}$ & 7 anos & Não & Não & 40,5 & 21 & 19 & 25 & - & - & 14 & 2 \\
\hline 30 & $\mathrm{M}$ & 6a $1 \mathrm{~m}$ & $7 \mathrm{a} 9 \mathrm{~m}$ & Não & Não & 39 & 14 & 63 & 63 & - & - & 20 & 1 \\
\hline 31 & $\mathrm{M}$ & $6 \mathrm{a} 10 \mathrm{~m}$ & $9 \mathrm{a} 9 \mathrm{~m}$ & Sim & Sim & 27,5 & 20 & 103 & 100 & - & - & 35 & 2 \\
\hline 21 & $\mathrm{M}$ & $8 \mathrm{a} 2 \mathrm{~m}$ & $8 \mathrm{a} 11 \mathrm{~m}$ & Não & Sim & 36 & 35 & 69 & 85 & - & - & 9 & 3 \\
\hline 32 & $\mathrm{M}$ & 12 a $5 \mathrm{~m}$ & $13 \mathrm{a} 5 \mathrm{~m}$ & Não & Não & - & 9 & 19 & 23 & - & - & 12 & 0 \\
\hline Médias & & $5 \mathrm{a} 6 \mathrm{~m}$ & $7 \mathrm{a}$ & $27,2 \%$ & $36,3 \%$ & 36,5 & 40,7 & 44,5 & 47,9 & 166 & 212,6 & 15,7 & 1,2 \\
\hline p-valor & & & & & 0,075 & 0,241 & $0,043^{*}$ & & & & & & \\
\hline
\end{tabular}


O PEP-R avalia tanto atraso no desenvolvimento como comportamentos típicos de autismo e oferece informações sobre sete áreas na Escala de Desenvolvimento: imitação, percepção, coordenação motora fina, coordenação motora grossa, integração olho mão, desenvolvimento cognitivo e cognitivo verbal, além de quatro áreas na Escala de Comportamento: linguagem, relacionamento e afeto, respostas sensoriais e interesses por materiais. Pode ser utilizado com crianças de 6 meses a 12 anos de idade. Foi adaptado e validado para a população brasileira por Leon, Bosa, Hugo e Hutz (2004). No presente estudo, foram utilizados os dados da Escala de Desenvolvimento (não os da Escala de Comportamento).

ACARS permite identificar crianças com características comportamentais de autismo e distinguir entre autismo e atraso no desenvolvimento sem autismo. De acordo com a escala, os resultados da avaliação podem ser distribuídos em três categorias: desenvolvimento normal (15-29,5), autismo leve/moderado (30-36,5) e autismo grave (acima de 37). Pode ser utilizada com crianças acima de 36 meses de idade. Essa escala foi adaptada e validada para a população brasileira por Pereira, Riesgo e Wagner (2008).

Os participantes com idades abaixo de 36 meses (P1, P2, P3, P4, P5 do Grupo 1 e P23 do Grupo 2) não foram avaliados pela CARS, pois esse instrumento não é destinado a avaliar menores de 36 meses. Os participantes com idades próximas ou acima de 6 anos (P14, P15, P16, P17, P18, P19, P20, P21, P22 do Grupo 1 e P28, P29, P30, P31, P32, P21 do Grupo 2) não foram avaliados pelo IPO, pois esse instrumento não é adequado para avaliar crianças acima de 6 anos. Todos os participantes foram avaliados pelo PEP-R.

\section{Procedimentos Adotados com o Grupo I (Intervenção Comportamental Intensiva)}

Inicialmente, as crianças foram avaliadas (pré-testes) por dois profissionais do CEI, um com formação em psicologia e o outro, em terapia ocupacional. Utilizou-se o PEP-R e o IPO para as crianças de 1 a 3 anos; entre 3 e 6 anos, o PEP-R, o IPO e a CARS; acima de 6 anos, o PEP-R e a CARS. O PEP-R foi o único instrumento comum a todos os participantes com autismo. A aplicação do PEP-R foi realizada em uma sala do CEI com poucos estímulos, em situação na qual um terapeuta apresentava as atividades e o outro registrava o desempenho da criança. O IPO foi aplicado na residência das crianças, em situação mais próxima do natural, a partir da observação do comportamento da criança e do relato dos cuidadores. A CARS foi preenchida pelos profissionais com base na observação do comportamento da criança. Foram necessárias, em média, duas semanas para a aplicação dos instrumentos de avaliação, análise dos dados e redação de relatório com os resultados de um participante. Após aproximadamente 12 meses, as crianças foram avaliadas novamente pelos mesmos instrumentos utilizados na avaliação inicial (pós-testes). Nem sempre a mesma dupla de terapeutas realizava a avaliação inicial e a final. Após a avaliação inicial, a intervenção era iniciada.

A intervenção era conduzida na residência das crianças com autismo, com destaque para a capacitação dos cuidadores. Dois profissionais, um psicólogo analista do comportamento e um terapeuta ocupacional, estavam presentes na residência dos participantes duas vezes por semana (cada profissional uma vez por semana), em sessões de uma hora cada. A função dos profissionais era ensinar os cuidadores a realizar as atividades com as crianças com autismo. Além disso, os profissionais também ensinavam os cuidadores a fazer registros sistemáticos do desempenho das crianças nas atividades (em protocolos elaborados pelo CEI, específicos para cada atividade) e supervisionavam a realização correta das atividades e dos registros. Os profissionais focavam na qualidade da execução das atividades, na qualidade dos registros dos cuidadores e na quantidade de atividades realizadas, para garantir que a intervenção fosse conduzida de maneira intensiva e criteriosa.

Para cada criança, era estabelecido um currículo de habilidades básicas, conforme Gomes e Silveira (2016), e um de autocuidados, baseado no IPO (Williams \& Aiello, 2001). A proposta de currículo era fundamentada nas necessidades identificadas para a criança e no conhecimento científico sobre condições ótimas para o ensino de cada tipo de habilidade, com os devidos esclarecimentos aos pais ou responsáveis, que contribuíam com decisões sobre prioridades. Os profissionais de psicologia eram responsáveis pela implementação de habilidades básicas e os de terapia ocupacional pelas de autocuidados. O currículo de habilidades básicas era dividido em cinco áreas: atenção, imitação, linguagem expressiva, linguagem receptiva e pré-acadêmica. Para cada área, o CEI desenvolveu programas de ensino em número variado, que se encontram detalhadamente descritos em Gomes e Silveira (2016), de modo a favorecer sua replicabilidade. Por exemplo, a área Habilidades de Imitação contava com os seguintes programas: imitar movimentos motores grossos, imitar ações com objetos, imitar movimentos motores finos, imitar movimentos fonoarticulatórios, imitar movimentos grossos em pé e imitar sequência de movimentos. O currículo de habilidades de autocuidados era dividido em quatro áreas: alimentação, higiene pessoal, vestuário e uso do banheiro, que englobavam habilidades variadas (e.g. higiene pessoal: escovar os dentes, tomar banho, vestir e despir, entre outras).

$\mathrm{O}$ ensino das habilidades especificadas nos currículos de habilidades básicas e de autocuidados era realizado pelos cuidadores com suas respectivas crianças, seguindo orientações sistemáticas dos profissionais especializados. As orientações abordavam tanto as condições de ensino (a preparação do ambiente e de materiais, como apresentar materiais e instruções, a exigência de resposta ativa, a apresentação de consequências identificadas como potencialmente reforçadoras, os critérios para concluir cada 
tarefa ou cada programa, entre outros aspectos), como o manejo geral dos comportamentos da criança. Para cada programa, eram especificados critérios de aprendizagem, uso de reforçadores, procedimentos de correção e número de habilidades treinadas por sessão.

Além disso, os cuidadores também registravam, em protocolos específicos para cada atividade, a data de realização da atividade, o número de tentativas de ensino e o desempenho da criança. Os protocolos utilizados eram simples (na maioria dos casos bastava marcar x para acertos ou erros), de modo a facilitar o registro dos cuidadores (Gomes \& Silveira, 2016). Os procedimentos de ensino eram fundamentados em Análise do Comportamento, porém nenhuma capacitação teórica foi realizada com os cuidadores, apenas orientações práticas e didáticas, com o uso preferencial pela modelação e uso mínimo de termos técnicos. Mais de um programa de ensino do currículo de habilidades básicas era inserido simultaneamente, dependendo da disponibilidade do cuidador e da criança. Em relação ao currículo de autocuidados, todas as habilidades eram ensinadas simultaneamente, isto é, ao longo de todo o período de intervenção, sempre articuladas com as situações de vida diária nas quais deveriam ocorrer.

\section{Coleta dos Dados}

A coleta de dados foi retrospectiva, realizada por meio das avaliações aplicadas pelos profissionais do CEI e dos protocolos de registro, preenchidos pelos cuidadores, referentes às atividades que eles realizavam com as crianças. Isto é, os pesquisadores trabalharam com os dados cujos registros foram realizados pelos profissionais do CEI e pelos cuidadores. A coleta e análise dos dados foram realizadas entre janeiro de 2015 e janeiro de 2016.

Os procedimentos empregados neste estudo foram aprovados pelo Comitê de Ética da Faculdade de Ciências Médicas de Minas Gerais (Parecer n. 923.913).

\section{Fidedignidade}

Dois avaliadores independentes e ingênuos quanto ao propósito da pesquisa contabilizaram avaliações dos Grupos 1 e 2 e protocolos de registro das atividades, preenchidos pelos cuidadores do Grupo 1. Essa contabilização foi realizada com os dados de 11 crianças selecionadas por sorteio (aproximadamente $30 \%$ do total; sete do Grupo 1 e quatro do Grupo 2) e o coeficiente de concordância (Kazdin, 1982) foi calculado. Para o Grupo 1, o cálculo do coeficiente considerou as variáveis idade e pontuação na CARS, PEP-R e IPO em pré e pós-testes, além do número de programas de ensino realizados. Para o Grupo 2, o cálculo do coeficiente considerou as variáveis idade e pontuação na CARS e no PEP-R na primeira e na segunda avaliação. O coeficiente de concordância encontrado para o Grupo 1 foi de $80 \%$ e para o Grupo 2 foi de $100 \%$. O coeficiente de concordância, considerando todas as variáveis dos dois grupos, foi de $87,5 \%$.

\section{RESULTADOS}

A Tabela 1 apresenta as características gerais dos participantes do Grupo 1. No início da intervenção, as crianças, das quais 20 meninos e duas meninas, tinham idades entre 1 ano e 3 meses e 11 anos e 4 meses. Seis eram falantes (em níveis variados de fala) e 16 não falavam. Todas apresentavam pontuação na CARS entre 26,5 (desenvolvimento normal) e 58 (autismo grave). É importante esclarecer que a CARS não é um instrumento de diagnóstico de autismo. Ela categoriza a criança em função da quantidade e da severidade dos sintomas. Dessa maneira, desenvolvimento normal não significa que a criança não tenha autismo, mas indica que a criança tem poucos sintomas de autismo. Os participantes do Grupo 1 fizeram entre 8 e 15 meses de intervenção intensiva, realizaram de 7 a 43 programas de ensino de habilidades básicas, além dos programas de habilidades de autocuidados, de 10 a 40 horas semanais de estimulação realizada pelos cuidadores (as horas citadas são estimativas baseadas nos registros e no relato dos cuidadores) e de nenhuma a 7 horas semanais de outras terapias (terapia ocupacional, fonoaudiologia ou musicoterapia) não intensivas. A aplicação dos pós-testes para esse grupo foi realizada entre 8 e 15 meses após os pré-testes.

Os participantes deste grupo exposto à intervenção intensiva tinham, em média, 4 anos e 8 meses no início da intervenção e 5 anos e 8 meses ao término; 27,2\% apresentavam fala no início e $54,5 \%$ apresentavam ao término. A pontuação média final e a inicial da CARS indicou diminuição dos sintomas de autismo de 40,5 (autismo grave) para 35,8 (autismo leve/moderado); a média no PEP-R indicou ganho de 9 meses no desenvolvimento (pontuação de 34,9 para 64,5) e no IPO indicou ganho de 12 meses (pontuação de 156,8 para 247,2 ). Um tratamento estatístico foi realizado por meio do teste de Wilcoxon (Magalhães \& Lima, 2009) para amostras pareadas, com o objetivo de verificar se havia diferenças significativas no desempenho dos participantes em pré e pós-testes. As diferenças foram consideradas significativas no nível de probabilidade menor do que $0,05(\mathrm{p}<0,05)$; acima desse valor, a hipótese nula de que as médias eram idênticas não seria rejeitada. Os resultados em todas as análises (CARS, PEP-R e IPO) indicaram valores de $\mathrm{p}$ menores do que 0,05 , ou seja, valores estatisticamente significativos para 
o Grupo 1. Os participantes desse grupo realizaram, em média, 11,8 meses de intervenção intensiva, com a execução de 17,6 programas de ensino em 15,2 horas semanais de intervenção intensiva e 1,5 horas semanais de outras terapias não intensivas.

A Tabela 2 apresenta as características gerais dos participantes do Grupo 2, que realizaram apenas as avaliações. Na primeira avaliação (A1), as crianças, das quais 9 meninos e duas meninas, tinham idades entre 2 anos 5 meses e 12 anos 5 meses. Três falavam (níveis variados de fala) e oito não falavam. Todas apresentavam pontuação na CARS entre 27,5 (desenvolvimento normal) e 45 (autismo grave). A aplicação da reavaliação ocorreu entre 9 e 35 meses após a primeira avaliação. Intervalos longos entre as avaliações se devem ao fato de que algumas crianças saíram do CEI e retornaram algum tempo depois, a critério de suas famílias.

Os participantes do Grupo 2 tinham, em média, 5 anos e 6 meses na primeira avaliação (A1) e 7 anos na reavaliação (A2). A pontuação final e a inicial da CARS indicou aumento dos sintomas de autismo de 36,5 (autismo leve/moderado) para 40,7 (autismo grave); o PEP-R não indicou ganhos no desenvolvimento, porém o IPO indicou ganhos de 7 meses no desenvolvimento (pontuação de 166 para 212,6). Os resultados das análises estatísticas realizadas por meio do teste de Wilcoxon para a CARS e o PEP-R indicaram valores de p maiores do que 0,05 , ou seja, valores não estatisticamente significativos, porém, para o IPO, indicaram diferença significativa com valor de $\mathrm{p}$ menor do que $0,05(\mathrm{p}=0,043)$.

Tratamento estatístico também foi realizado por meio do teste de Wilcoxon para amostras pareadas, com o objetivo de verificar se havia diferença significativa no desempenho dos participantes por áreas do desenvolvimento no PEP-R
(Tabela 3) e no IPO (Tabela 4). Em relação ao desempenho dos participantes com autismo por áreas do PEP-R, observam-se diferenças estatisticamente significativas em todas as áreas para o Grupo 1, e apenas em Percepção e Coordenação Motora Fina para o Grupo 2. Com relação ao IPO, a diferença foi significativa em todas as áreas para o Grupo 1, e apenas em Autocuidados no Grupo 2.

A Figura 1 apresenta as médias de ganhos no desenvolvimento (em meses) dos participantes com autismo dos Grupos 1 e 2, por áreas no PEP-R e no IPO. No PEP-R, os participantes do Grupo 1 apresentaram médias superiores às do Grupo 2 em todas as áreas do desenvolvimento. Ganhos próximos ou acima de 12 meses ocorreram em imitação, percepção, coordenação motora fina e integração olho-mão. Ganhos mais baixos foram observados em coordenação motora grossa, desenvolvimento cognitivo e cognitivo verbal. No IPO, os participantes do Grupo 1 também apresentaram ganhos médios mais altos do que os do Grupo 2 na maioria das áreas, exceto em linguagem. Apesar da média do Grupo 2 em linguagem ter sido superior à do Grupo 1, o valor não foi estatisticamente significativo. Ganhos próximos ou acima de 12 meses foram observados na maioria das áreas do desenvolvimento para o Grupo 1, exceto em linguagem.

Foi analisada a relação entre o ganho de habilidades dos participantes com autismo do Grupo 1 no PEP-R (diferença entre a pontuação final e a inicial) e as seguintes variáveis: pontuação na CARS, idade dos participantes com autismo no início da intervenção, número de programas de ensino realizados e horas semanais de intervenção intensiva. O coeficiente de correlação linear de Pearson foi calculado. Os dados indicaram baixa correlação entre o desempenho no PEP-R e o número de programas $(r=0,15)$ e horas semanais de intervenção $(r=-0,15)$. Porém,

Tabela 3

Médias, Interpretação da Pontuação, Diferenças e p-valor dos Participantes com Autismo do Grupo 1 (Porção Superior) e do Grupo 2 (Porção Inferior) por Área do Desenvolvimento no PEP-R: Imitação, Percepção, Motor Fina, Motora Grossa, Integração Olho-Mão, Desempenho Cognitivo e Desempenho Cognitivo-Verbal.

\begin{tabular}{|c|c|c|c|c|c|c|c|c|c|c|c|c|c|c|}
\hline \multicolumn{15}{|c|}{ GRUPO 1} \\
\hline & \multicolumn{2}{|c|}{ Imitação } & \multicolumn{2}{|c|}{ Percepção } & \multicolumn{2}{|c|}{ Motora F. } & \multicolumn{2}{|c|}{ Motora G. } & \multicolumn{2}{|c|}{ Olho-mão } & \multicolumn{2}{|c|}{ D. Cognitivo } & \multicolumn{2}{|c|}{ D. C. Verbal } \\
\hline & Pré & Pós & Pré & Pós & Pré & Pós & Pré & Pós & Pré & Pós & Pré & Pós & Pré & Pós \\
\hline Média & 3,3 & 9,5 & 7,3 & 10,1 & 6,7 & 10,7 & 10,2 & 14,6 & 3,6 & 7,4 & 2,8 & 7,7 & 1 & 4,4 \\
\hline Interpretação & $1 \mathrm{a} 1 \mathrm{~m}$ & $2 \mathrm{a} 4 \mathrm{~m}$ & $1 \mathrm{a} 6 \mathrm{~m}$ & $2 \mathrm{a} 7 \mathrm{~m}$ & 1a $8 \mathrm{~m}$ & 2a $8 \mathrm{~m}$ & 1a $8 \mathrm{~m}$ & $2 \mathrm{a} 6 \mathrm{~m}$ & 1a $9 \mathrm{~m}$ & $3 \mathrm{a} 1 \mathrm{~m}$ & $1 \mathrm{a}$ & 1a $8 \mathrm{~m}$ & 1a $5 \mathrm{~m}$ & 2a $1 \mathrm{~m}$ \\
\hline Diferença & \multicolumn{2}{|c|}{$15 \mathrm{~m}$} & \multicolumn{2}{|c|}{$13 \mathrm{~m}$} & \multicolumn{2}{|c|}{$12 \mathrm{~m}$} & \multicolumn{2}{|c|}{$10 \mathrm{~m}$} & \multicolumn{2}{|c|}{$16 \mathrm{~m}$} & \multicolumn{2}{|c|}{$8 \mathrm{~m}$} & \multicolumn{2}{|c|}{$8 \mathrm{~m}$} \\
\hline p-valor & \multicolumn{2}{|c|}{$0,000 *$} & \multicolumn{2}{|c|}{$0,000^{*}$} & \multicolumn{2}{|c|}{$0,000^{*}$} & \multicolumn{2}{|c|}{$0,000 *$} & \multicolumn{2}{|c|}{$0,000^{*}$} & \multicolumn{2}{|c|}{$0,000^{*}$} & \multicolumn{2}{|c|}{$0,001 *$} \\
\hline \multicolumn{15}{|c|}{ GRUPO 2} \\
\hline & \multicolumn{2}{|c|}{ Imitação } & \multicolumn{2}{|c|}{ Percepção } & \multicolumn{2}{|c|}{ Motora F. } & \multicolumn{2}{|c|}{ Motora G. } & \multicolumn{2}{|c|}{ Olho-mão } & \multicolumn{2}{|c|}{ D. Cognitivo } & \multicolumn{2}{|c|}{ D. C. Verbal } \\
\hline & A1 & A2 & A1 & A2 & A1 & A2 & A1 & A2 & A1 & A2 & A1 & A2 & $\mathbf{A 1}$ & A2 \\
\hline Média & 5,4 & 5,5 & 7 & 8,8 & 8,1 & 9,4 & 11,4 & 11,3 & 4,5 & 5,3 & 5,4 & 5,1 & 2,1 & 2,6 \\
\hline Interpretação & $1 \mathrm{a} 6 \mathrm{~m}$ & $1 \mathrm{a} 6 \mathrm{~m}$ & 1a $5 \mathrm{~m}$ & $2 \mathrm{a}$ & $2 \mathrm{a} 1 \mathrm{~m}$ & $2 \mathrm{a} 4 \mathrm{~m}$ & $\begin{array}{c}1 \mathrm{a} \\
11 \mathrm{~m}\end{array}$ & $\begin{array}{c}1 \mathrm{a} \\
11 \mathrm{~m}\end{array}$ & $2 \mathrm{a} 2 \mathrm{~m}$ & $2 \mathrm{a} 5 \mathrm{~m}$ & 1a $4 \mathrm{~m}$ & 1a $3 \mathrm{~m}$ & 1a $8 \mathrm{~m}$ & 1a $9 \mathrm{~m}$ \\
\hline Diferença & \multicolumn{2}{|c|}{0} & $7 \mathrm{~m}$ & & \multicolumn{2}{|l|}{$3 \mathrm{~m}$} & \multicolumn{2}{|c|}{0} & \multicolumn{2}{|c|}{$3 \mathrm{~m}$} & \multicolumn{2}{|c|}{0} & & \\
\hline p-valor & & & $0,011 *$ & & $0,016^{*}$ & & & & & & & & & \\
\hline
\end{tabular}

Nota: o asterisco indica diferenças estatisticamente significativas $(\mathrm{p}<0,05)$, Teste de Wilcoxon 
Tabela 4

Médias, Interpretação da Pontuação, Diferenças e p-valor dos Participantes com Autismo do Grupo 1 (Porção Superior) e do Grupo 2 (Porção Inferior) por Área do Desenvolvimento no IPO: Autocuidados, Cognição, Desenvolvimento Motor, Linguagem e Socialização.

\begin{tabular}{|c|c|c|c|c|c|c|c|c|c|c|}
\hline \multicolumn{11}{|c|}{ GRUPO 1} \\
\hline & \multicolumn{2}{|c|}{ Autocuidados } & \multicolumn{2}{|c|}{ Cognição } & \multicolumn{2}{|c|}{ D. Motor } & \multicolumn{2}{|c|}{ Linguagem } & \multicolumn{2}{|c|}{ Socialização } \\
\hline & Pré & Pós & Pré & Pós & Pré & Pós & Pré & Pós & Pré & Pós \\
\hline Média & 24,1 & 50,8 & 18,5 & 38,2 & 79,4 & 94,8 & 6,9 & 16,6 & 28,6 & 43,9 \\
\hline Interpretação & 1a $8 \mathrm{~m}$ & $3 a$ & 1a $7 \mathrm{~m}$ & 2a $7 \mathrm{~m}$ & 2a $11 \mathrm{~m}$ & 3a $10 \mathrm{~m}$ & $7 \mathrm{~m}$ & $1 \mathrm{a} 2 \mathrm{~m}$ & $11 \mathrm{~m}$ & $2 \mathrm{a} 3 \mathrm{~m}$ \\
\hline Diferença & \multicolumn{2}{|c|}{$16 \mathrm{~m}$} & \multicolumn{2}{|c|}{$12 \mathrm{~m}$} & \multicolumn{2}{|c|}{$11 \mathrm{~m}$} & \multicolumn{2}{|c|}{$7 \mathrm{~m}$} & \multicolumn{2}{|c|}{$16 \mathrm{~m}$} \\
\hline p-valor & \multicolumn{2}{|c|}{$0,001^{*}$} & \multicolumn{2}{|c|}{$0,002 *$} & \multicolumn{2}{|c|}{$0,001 *$} & \multicolumn{2}{|c|}{$0,005^{*}$} & \multicolumn{2}{|c|}{$0,003 *$} \\
\hline \multicolumn{11}{|c|}{ GRUPO 2} \\
\hline & \multicolumn{2}{|c|}{ Autocuidados } & \multicolumn{2}{|c|}{ Cognição } & \multicolumn{2}{|c|}{ D. Motor } & \multicolumn{2}{|c|}{ Linguagem } & \multicolumn{2}{|c|}{ Socialização } \\
\hline & A1 & A2 & A1 & A2 & A1 & $\mathbf{A 2}$ & A1 & A2 & A1 & A2 \\
\hline Média & 27,2 & 36,8 & 23 & 26,4 & 81,2 & 91,8 & 9 & 22 & 25,2 & 40 \\
\hline Interpretação & 1a $11 \mathrm{~m}$ & $2 \mathrm{a} 4 \mathrm{~m}$ & 1a $10 \mathrm{~m}$ & $2 a$ & $3 a$ & $3 a \mathrm{~m}$ & $10 \mathrm{~m}$ & $1 \mathrm{a} 6 \mathrm{~m}$ & $9 \mathrm{~m}$ & $1 \mathrm{a} 11 \mathrm{~m}$ \\
\hline Diferença & \multicolumn{2}{|c|}{$5 \mathrm{~m}$} & \multicolumn{2}{|c|}{$2 \mathrm{~m}$} & \multicolumn{2}{|c|}{$8 \mathrm{~m}$} & \multicolumn{2}{|c|}{$8 \mathrm{~m}$} & \multicolumn{2}{|c|}{$14 \mathrm{~m}$} \\
\hline p-valor & \multicolumn{2}{|c|}{$0,043 *$} & \multicolumn{2}{|c|}{0,336} & \multicolumn{2}{|c|}{0,138} & \multicolumn{2}{|c|}{0,273} & \multicolumn{2}{|c|}{0,138} \\
\hline
\end{tabular}

Nota: $\mathrm{o}$ asterisco indica diferenças estatisticamente significativas $(\mathrm{p}<0,05)$

\section{IPO}

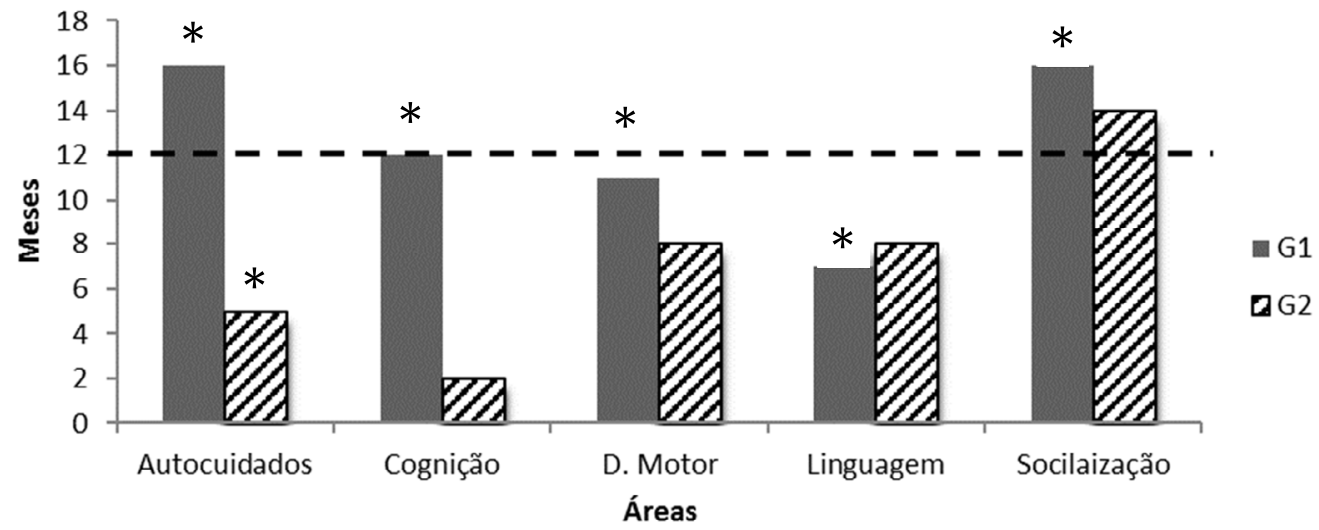

\section{PEP-R}

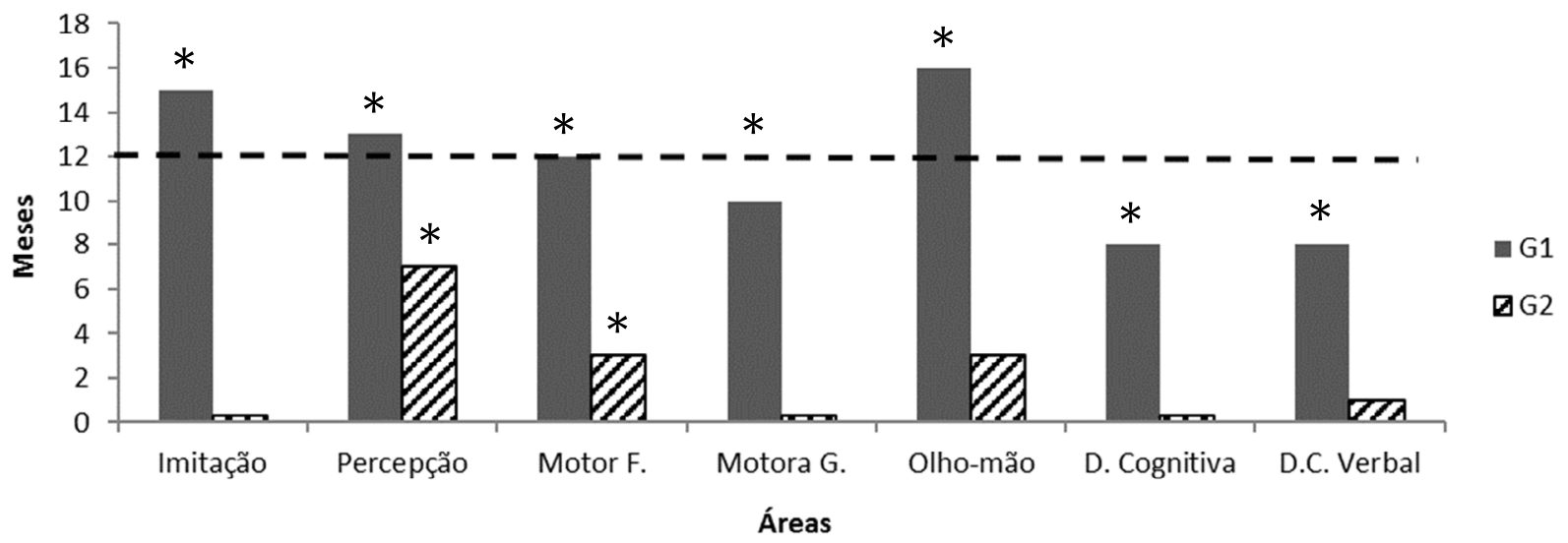

Figura 1. Média de ganhos no desenvolvimento (em meses) dos participantes com autismo dos Grupos 1 (com intervenção) e 2 (sem a mesma intervenção), por área do desenvolvimento no PEP-R (porção superior) e no IPO (porção inferior). Asterisco indica valores estatisticamente significativos. A linha tracejada indica 12 meses no desenvolvimento. 
indicou correlação média entre o desempenho no PEP-R e a pontuação na CARS $(\mathrm{r}=-0,54)$, assim como em relação à idade dos participantes $(\mathrm{r}=-0,56)$. De maneira geral, quanto maior o grau de autismo e a idade da criança, menor o ganho.

Outro aspecto analisado foi a média de ganhos de habilidades dos participantes com autismo do Grupo 1 no PEP-R (diferença entre a pontuação final e a inicial), quando distribuídos em falantes e não falantes (ao início da intervenção) e por faixa etária (entre 0 e 3 anos; entre 3 anos e 1 mês e 5 anos; e acima de 5 anos). Os falantes ganharam aproximadamente 7 meses a mais no desenvolvimento do que os não falantes. Além disso, o ganho diminuiu à medida que a idade aumentou. Em todas as situações, as diferenças foram estatisticamente significativas, de acordo com o teste de Wilcoxon (Tabela 5 e Figura 2).

Tabela 5

Pontuação Média e Diferença no Desempenho dos Participantes do Grupo 1 no PEP-R, Divididos em Falantes e não Falantes e por Faixa Etária; 0 a 3 Anos; 3 Anos e 1 Mês a 5 Anos; Acima de 5 Anos e 1 Mês

\begin{tabular}{|c|c|c|c|c|c|c|c|c|c|c|}
\hline & \multicolumn{2}{|c|}{ Falantes } & \multicolumn{2}{|c|}{ Não-Falantes } & \multicolumn{2}{|c|}{0 a $3 a$} & \multicolumn{2}{|c|}{$3 a 1 m$ a $5 a$} & \multicolumn{2}{|c|}{$>$ que $5 \mathrm{a} 1 \mathrm{~m}$} \\
\hline & Pré & Pós & Pré & Pós & Pré & Pós & Pré & Pós & Pré & Pós \\
\hline Média & 54 & 85,8 & 27,8 & 56,6 & 18,8 & 56 & 40,9 & 71,8 & 38,6 & 62,9 \\
\hline Interpretação & 1a $10 \mathrm{~m}$ & $3 \mathrm{a}$ & 1a $4 \mathrm{~m}$ & $1 \mathrm{a} 11 \mathrm{~m}$ & 1a $1 \mathrm{~m}$ & 1a $11 \mathrm{~m}$ & 1a $7 \mathrm{~m}$ & $2 \mathrm{a} 4 \mathrm{~m}$ & $1 \mathrm{a} 6 \mathrm{~m}$ & 2a $1 \mathrm{~m}$ \\
\hline Diferença & \multicolumn{2}{|c|}{$14 \mathrm{~m}$} & \multicolumn{2}{|c|}{$7 \mathrm{~m}$} & \multicolumn{2}{|c|}{$10 \mathrm{~m}$} & \multicolumn{2}{|c|}{$9 m$} & \multicolumn{2}{|c|}{$7 \mathrm{~m}$} \\
\hline p-valor & \multicolumn{2}{|c|}{$0,028^{*}$} & \multicolumn{2}{|c|}{$0,000^{*}$} & \multicolumn{2}{|c|}{$0,043 *$} & \multicolumn{2}{|c|}{$0,012 *$} & \multicolumn{2}{|c|}{$0,008^{*}$} \\
\hline
\end{tabular}

Nota: $\mathrm{o}$ asterisco indica diferenças estatisticamente significativas $(\mathrm{p}<0,05)$

\section{PEP-R}

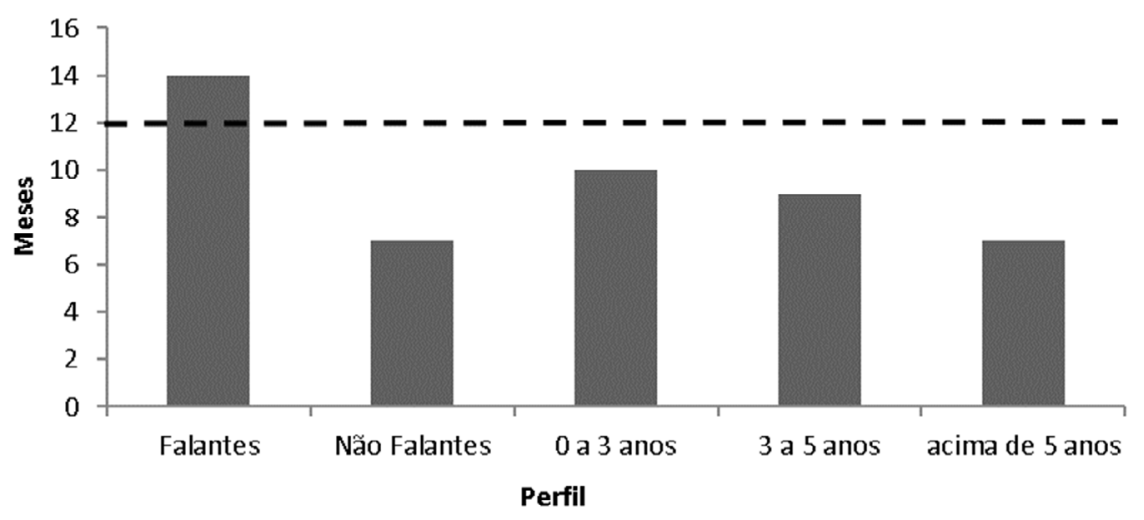

\section{Cuidador}

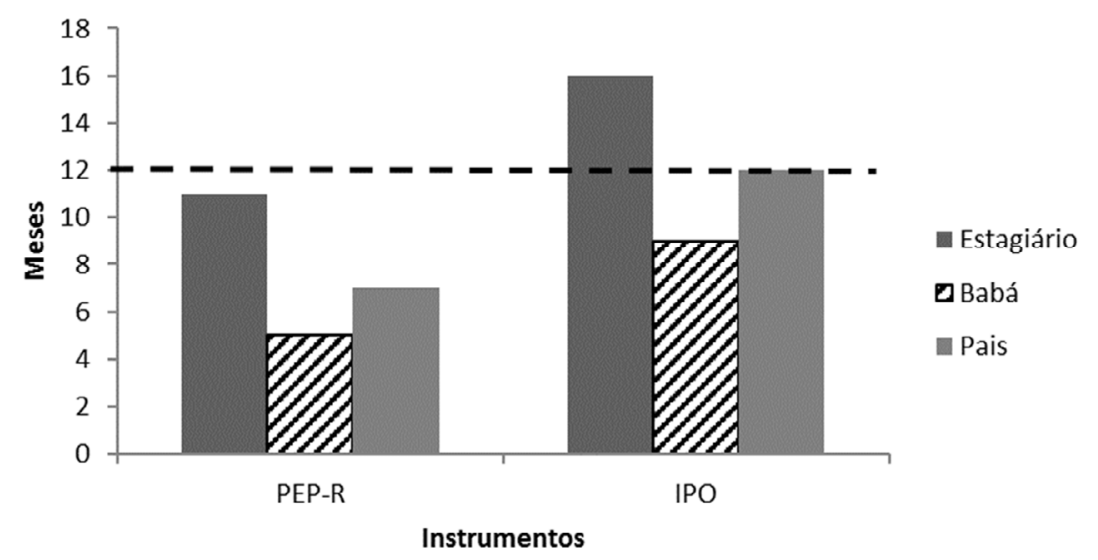

Figura 2. Média de ganhos no desenvolvimento (em meses) dos participantes com autismo do Grupo 1, considerando-se a presença ou ausência de fala ou idade no início do estudo (porção superior) e no PEP-R e no IPO, quando a intervenção foi realizada por estagiários, babás ou pais (porção inferior). A linha tracejada indica 12 meses no desenvolvimento. 
O desempenho do Grupo 1 também foi analisado considerando-se a variável cuidador (estagiários, babás ou pais). As crianças que fizeram a intervenção com estagiários apresentaram ganhos maiores no desenvolvimento, seguidas das crianças que a realizaram com os pais. Ganhos mais baixos foram observados com as crianças que realizaram a intervenção com as babás (Figura 2).

De maneira geral, os dados sugerem que a ICI realizada por cuidadores, sob a orientação de profissionais especializados, promoveu ganhos significativos em todas as áreas do desenvolvimento das crianças com autismo que passaram pelo primeiro ano de ICI. Enquanto isso, as crianças do grupo sem a mesma intervenção apresentaram ganhos menos expressivos. Além disso, os dados indicaram que a presença ou não de fala, o grau de autismo, a idade das crianças e o tipo de cuidador foram variáveis relevantes para os efeitos da intervenção.

\section{DISCUSSÃO}

Este estudo foi baseado em dados retrospectivos do primeiro ano de ICI realizada por cuidadores com um grupo de crianças com autismo. Seu objetivo foi avaliar os efeitos desse tipo de intervenção no desenvolvimento das crianças e comparar o desempenho dessas crianças com outras que não fizeram a intervenção intensiva.

Os dados gerais indicaram ganhos significativos em todas as áreas do desenvolvimento das crianças com autismo que passaram pelo primeiro ano de ICI, enquanto as crianças do outro grupo apresentaram ganhos menos expressivos em algumas dessas áreas. Os resultados são condizentes com a literatura que indica efeitos melhores desse tipo de intervenção, quando comparados aos efeitos de outros tipos de terapias ou de terapias não intensivas (Lovaas, 1987; Sherman et al., 1988; Smith, 1999; Warren et al., 2011). Por outro lado, a literatura indica que a ICI deve ser realizada por dois anos ou mais (Green, 1996), razão pela qual será importante dar continuidade ao estudo, de modo a avaliar, periódica e cumulativamente, os efeitos de longo prazo.

As crianças mais novas, com baixa pontuação na CARS (sintomas mais brandos de autismo) e que apresentavam fala no início do estudo obtiveram ganhos maiores no desenvolvimento. Esses resultados condizem com a literatura sobre ICI (e.g., Beglinger \& Smith, 2005; Harris \& Handleman, 2000; Luiselli et al., 2000). Por exemplo, Dawson et al. (2010), Eikeseth et al. (2002) e Green (1996) mostraram que a idade da criança no início da intervenção é uma variável importante, de modo que melhores resultados são obtidos com crianças mais novas, antes dos 4 anos de idade. Weiss (1999) indicou que a pontuação na CARS também poderia ser uma variável importante, porém Harris e Handleman (2000) não observaram uma relação direta entre a pontuação na CARS e os efeitos da intervenção intensiva. Em relação à fala, Green (1996) ressaltou que crianças com mais habilidades de linguagem apresentam maiores ganhos. Outros aspectos importantes sugeridos pela literatura (Harris \& Handleman, 2000; Weiss, 1999) são a taxa de aprendizagem inicial e o Coeficiente de Inteligência (QI). Porém, essas variáveis não foram medidas no presente estudo.

As avaliações utilizadas neste trabalho também são um ponto importante a ser discutido. Como o autismo é um transtorno do desenvolvimento, medidas que indiquem quais áreas do desenvolvimento estão alteradas e atrasadas e as dimensões desse atraso podem ser importantes para caracterizar o repertório da criança e para planejar e avaliar os efeitos da intervenção (Schopler et al., 1990). Um indicador fundamental dos efeitos da ICI é o quanto o desenvolvimento da criança com autismo se aproxima do desenvolvimento de uma criança com desenvolvimento típico, sem autismo, em todas as áreas. Alguns estudos usaram medidas de QI associadas a medidas do desenvolvimento, semelhantes às que foram usadas no presente estudo (Anderson et al., 1987; Luiselli et al., 2000; Sheinkopf \& Sielgel, 1998; Sherman et al., 1988; Weiss, 1999) e outros utilizaram a medida de QI associada a medidas que avaliaram o tipo de escola ou nível de suporte escolar, após o término da intervenção intensiva, como parâmetros para avaliar os efeitos da intervenção (Boyd \& Corley, 2001; Lovaas, 1987; Reed et al., 2007; Smith et al., 2000). O tipo de escola ou nível de suporte escolar pode ser uma medida importante para sistemas educacionais nos quais a criança começa a escolarização com grande suporte - como, por exemplo, em instituições especializadas - e gradativamente passa a frequentar ou não escolas comuns (regulares), com ou sem apoio, dependendo do nível do seu desenvolvimento. No Brasil, o sistema educacional prioriza a escolarização em escolas comuns, com o apoio de estagiários, mediadores e professores auxiliares; e, atualmente, a maioria das crianças com autismo, independentemente do grau de atraso no desenvolvimento, inicia sua vida escolar nesse tipo de instituição, sem que haja a diminuição gradativa dos níveis de suporte (Gomes \& Mendes, 2010; Mendes, 2006). Dessa maneira, o nível de suporte escolar pode não ser uma boa medida para avaliar os efeitos da intervenção intensiva no Brasil.

As crianças que fizeram a intervenção com estagiários apresentaram resultados melhores do que aquelas que a realizaram com os pais ou com as babás. Contudo, essa diferença tem que ser considerada com muito cuidado, tendo em vista a complexidade da situação e as muitas variáveis que podem estar envolvidas. Esse resultado pode ser efeito da maneira como os cuidadores foram orientados, assim como de variáveis dos familiares e das babás. O estresse familiar, 
que afeta frequentemente muitas famílias de pessoas com autismo, pode ter influenciado o desempenho dos pais, por exemplo (Fávero \& Santos, 2005). As atividades rotineiras das babás no cuidado com as crianças, além das atividades orientadas pelos terapeutas, podem ter comprometido seu desempenho na intervenção intensiva. $\mathrm{O}$ fato de as crianças terem apresentado desempenhos mais baixos com pais e babás é um dado importante, porém não deve ser visto como uma limitação para a realização da intervenção intensiva com esses cuidadores; ao contrário, deve ser visto como um indicador da necessidade de se capacitar melhor esses cuidadores, pois a literatura indica os benefícios dessa capacitação para garantir a manutenção e generalização dos resultados, além de diminuir o estresse familiar e os custos com a intervenção (Green, 1996; McConachie \& Diggle, 2006).

Outro aspecto importante se refere às horas de intervenção; apesar da média de 15 horas semanais realizadas pelo Grupo 1, sete crianças desse grupo realizaram 10 horas semanais (P1, P3, P6, P9, P11, P16 e P20), situandose abaixo do que a literatura indica como intensiva (15 a 40 horas semanais). Além disso, os dados não indicaram uma correlação entre o número de horas e o desempenho dos participantes e nem entre o desempenho e o número de programas de ensino. Nesse sentido, os resultados confirmam os de Luiselli et al. (2014), que indicaram ganhos no desenvolvimento de participantes expostos, em média, a 11,8 horas de intervenção por semana. Esses pesquisadores perceberam uma relação maior entre o desempenho e o tempo de intervenção (meses) do que entre o desempenho e as horas semanais de intervenção e ressaltaram a importância de se diferirem a quantidade de horas semanais e as oportunidades que a criança tem de aprender habilidades novas. Dessa maneira, horas de intervenção não significa necessariamente que a criança está tendo a intervenção adequada; variáveis relacionadas à estrutura da intervenção, à orientação aos cuidadores, aos procedimentos de ensino, correção, manutenção e generalização podem comprometer os resultados. Por outro lado, a Análise do Comportamento, como outras ciências, mudou muito desde a década de 1980, quando se considerava a necessidade de 40 horas semanais de intervenção (Lovaas, 1987), até a atualidade (Cruz, 2006). Procedimentos que eram bastante utilizados naquela época, como a punição, deram lugar a estratégias mais eficazes de controle de estímulos e de reforçamento (Sidman, 1995). Apesar de resultados como os relatados no presente estudo indicarem ganhos no desenvolvimento com intervenções realizadas em 10 horas semanais, é importante ressaltar que isso também não indica necessariamente que intervenções "menos intensivas" produzam os mesmos resultados que as "intervenções mais intensivas"; essa é uma questão metodológica que deve continuar sendo investigada, inclusive com um controle mais preciso das horas de intervenção.

Os dados das crianças do Grupo 2 indicam ganhos pouco expressivos, condizentes com a literatura que indica que o autismo é crônico e que intervenções não intensivas não produzem efeitos significativos no desenvolvimento (Lovaas, 1987; Sherman et al., 1988; Smith, 1999; Warren et al., 2011). Além disso, os dados desse grupo apontam e reforçam a efetividade da intervenção realizada com o Grupo 1.

Os resultados do estudo são animadores por indicarem melhora expressiva no desenvolvimento das crianças brasileiras que realizaram a intervenção, porém esbarra em problemas metodológicos comuns em pesquisas semelhantes, relacionados às dificuldades operacionais e éticas em distribuir randomicamente os participantes entre grupo experimental e controle, o tempo de intervenção (que pode durar dois ou três anos), as horas semanais de terapia, os custos e as dificuldades de controle de variáveis, principalmente em intervenções realizadas por cuidadores e em ambiente domiciliar (Smith, 1999; Smith et al., 2000; Warren et al., 2011). Apesar das dificuldades e da falta de controle experimental rigoroso, os resultados alcançados tiveram magnitude relevante no contexto dos participantes. Isto sugere que, apesar das dificuldades de controle experimental, os programas estruturados de ensino, mesmo que aplicados sem a desejável integridade, devem conter variáveis críticas, poderosas o suficiente para competir com um grande número de variáveis intervenientes no ambiente e na aplicação da intervenção e gerar mudanças relevantes no repertório dos participantes. Essa constatação não reduz a importância de investigações sistemáticas e rigorosas que ampliem o conhecimento a respeito de estratégias efetivas para o tratamento do autismo; pelo contrário, sugere a possibilidade de que, com maior controle e rigor, os resultados possam ser ainda mais expressivos.

\section{REFERÊNCIAS}

Aiello, A. L. R. (2002). Identificação precoce de sinais de autismo. In H. J. Guilhardi, M. B. B. P. Madi, P. P. Queiroz, \& M. C. Scoz. (Orgs.), Sobre comportamento e cognição: Contribuições para a construção da Teoria do Comportamento (pp. 13-29). Santo André, SP: ESETec.

Anderson, S. R., Avery, D. L., DiPietro, E. K., Edwards, G. L., \& Christian, W. P. (1987). Intensive home-based early intervention with autistic children. Education and Treatment of Children, 10, 352-366.
Associação Americana de Psiquiatria. (2002). Manual diagnóstico e estatístico de transtornos mentais ( $4^{\mathrm{a}} \mathrm{ed}$. Rev). Porto Alegre: Artes Médicas.

Associação Americana de Psiquiatria. (2013). Diagnostic and statistical manual of mental disorders ( $5^{\mathrm{a}}$ ed.). Washington: American Psychiatric Association.

Baer, D. M., Wolf, M. M., \& Risley, T. R. (1987). Some still-current dimensions of applied behavior analysis. Journal of Applied Behavior Analysis, 20(4), 91-97. 
Beglinger, L., \& Smith, T. (2005). Concurrent validity of social subtype and IQ after early intensive behavioral intervention in children with autism: A preliminary investigation. Journal of Autism and Developmental Disorders, 35(3), 295-303.

Bluma, S., Shearer, M., Frohman, A., \& Hilliard, J. (1976). Portage Guide to Early Education Manual (Ed. Rev.). Portage, Wisconsin: CESA-12.

Boyd, R. D., \& Corley, M. J. (2001). Outcome survey of early intensive behavioral intervention for young children with autism in a community setting. Autism, 5(4), 430-441.

Campbell, M., Schopler, E., Cueva, J., \& Hallin, A. (1996). Treatment of autistic disorder. Journal of the American Academy of Child \& Adolescent Psychiatry, 35(2), 134-143.

Cruz, R. N. (2006). História e historiografia da ciência: Considerações para pesquisa histórica em Análise do Comportamento. Revista Brasileira de Terapia Comportamental e Cognitiva, 8(2), 161-178.

Dawson, G., Rogers, S., Munson, J., Smith, M., Winter, J., Greenson, J., ... Varley, J. (2010). Randomized, controlled trial of an intervention for toddlers with autism: The Early Start Denver Model. Pediatrics, 125(1), 17-23.

Dawson, G., \& Osterling, J. (1997). Early intervention in autism: Effectiveness and common elements of current approaches. In M. J. Guralnick (Ed.), The effectiveness of early intervention: Second generation research (pp. 307-326). Baltimore: Brookes.

Eikeseth, S., Smith, T., Jahr, E., \& Eldevik, S. (2002). Intensive behavioral treatment at school for 4-to 7-year-old children with autism: A 1-year comparison controlled study. Behavior Modification, 26(1), 49-68.

Fávero, M. Â. B., \& Santos, M. D. (2005). Autismo infantil e estresse familiar: Uma revisão sistemática da literatura. Psicologia: Reflexão e Crítica, 18(3), 358-369.

Gargiulo, R. M. (2006). Special education in contemporary society: An introduction to exceptionality. Belmont, CA: Wadsworth/ Thomson Learning.

Gomes, C. G. S., \& Mendes, E. G. (2010). Escolarização inclusiva de alunos com autismo na rede municipal de ensino de Belo Horizonte. Revista Brasileira de Educação Especial, 16(3), 375-396.

Gomes, C. G. S., \& Silveira, A. D. (2016). Ensino de habilidades básicas para pessoas com autismo: Manual para Intervenção Comportamental Intensiva. Curitiba: Appris.

Green, G. (1996). Early behavioral intervention for autism: What does research tell us? In C. Maurice, G. Green, \& S. Luce (Eds.), Behavioral intervention for young children with autism: A manual for parents and professionals (pp. 29-44). Austin (TX): Pro-Ed.

Gresham, F. M., \& MacMillan, D. L. (1998). Early intervention project: Can its claims be substantiated and its effects replicated? Journal of Autism and Developmental Disorders, $28,5-13$.

Harris, S. L., \& Handleman, J. S. (2000). Age and IQ at intake as predictors of placement for young children with autism: A fourto six-year follow-up. Journal of Autism and Developmental Disorders, 30(2), 137-142.

Kazdin, A. E. (1982). Single-case research designs: Methods for clinical and applied settings. New York: Oxford University Press.

Leon, V. C., Bosa, C. A., Hugo, C. N., \& Hutz, C. (2004). Propriedades psicométricas do Perfil Psicoeducacional Revisado: PEP-R. Avaliação Psicológica, 3(1), 39-52.

Lovaas, O. I. (1987). Behavioral treatment and normal educational and intellectual functioning in young autistic children. Journal of Consulting and Clinical Psychology, 55(1), 3-9.

Luiselli, J. K., Cannon, B. O. M., Ellis, J. T., \& Sisson, R. W. (2000). Home-based behavioral intervention for young children with autism/pervasive developmental disorder: A preliminary evaluation of outcome in relation to child age and intensity of service delivery. Autism, 4(4), 426-438.
Magalhães, M. N., \& Lima, A. C. P. (2009). Noções de probabilidade estatística. São Paulo: Edusp.

Maurice, C. (1993). Let me hear your voice. New York: Knopf.

McConachie, H., \& Diggle, T. (2007). Parent implemented early intervention for young children with autism spectrum disorder: A systematic review. Journal of Evaluation in Clinical Practice, 13(1), 120-129.

McEachin, J. J., Smith, T., \& Lovaas, O. I. (1993). Long-term outcome for children with autism who received early intensive behavioral treatment. American Journal on Mental Retardation, 97(4), 359-372.

Mendes, E. G. (2006). A radicalização do debate sobre inclusão escolar no Brasil. Revista Brasileira de Educação, 11(33), 387-405.

Organização Mundial de Saúde. (1993). Classificação de transtornos mentais e de comportamentos da CID-10: Descrições clínicas e diretrizes diagnósticas. Porto Alegre: Artes Médicas.

Pereira, A., Riesgo, R. S., \& Wagner, M. B. (2008). Autismo infantil: Tradução e validação da Childhood Autism Rating Scale para uso no Brasil. Jornal de Pediatria, 84(6), 487-494.

Reed, P., Osborne, L. A., \& Corness, M. (2007). Brief report: Relative effectiveness of different home-based behavioral approaches to early teaching intervention. Journal of Autism and Developmental Disorders, 37(9), 1815-1821.

Schopler, E., Reichler, J. R., \& Renner, C. (1988). CARS - The Childhood Autism Rating Scale. Los Angeles: Westerm Pychological Services.

Schopler, E., Reichler, R. J., Bashford, A., Lansing, M. D., \& Marcus, L. M. (1990). Individualized assessment and treatment for autistic and developmentally disabled children: Psychoeducational Profile-Revised (PEP-R). Austin (TX): Pro- Ed.

Schopler, E., Short, A., \& Mesibov, G. (1989). Relation of behavioral treatment to normal functioning: Comment on Lovaas. Journal of Consulting and Clinical Psychology, 57, 162-164.

Sheinkopf, S. J., \& Siegel, B. (1998). Home-based behavioral treatment of young children with autism. Journal of Autism and Developmental Disorders, 28(1), 15-23.

Sherman, J., Barker, P., Lorimer, P., Swinson, R., \& Factor, D. C. (1988). Treatment of autistic children: Relative effectiveness of residential, out-patient and home-based interventions. Child Psychiatry and Human Development, 19(2), 109-125.

Sidman, M. (1995). Coerção e suas implicações. Campinas: Editora Livro Pleno.

Skinner, B. F. (1953). Science and human behavior. New York: McMillan.

Skinner, B. F. (1968). The technology of teaching. New York: Appleton-CenturyCrofts.

Smith, T. (1999). Outcome of early intervention for children with autism. Clinical Psychology: Science and Practice, 6(1), 33-49.

Smith, T., Buch, G. A., \& Gamby, T. E. (2000). Parent-directed, intensive early intervention for children with pervasive developmental disorder. Research in Developmental Disabilities, 21(4), 297-309.

Valenti, M., Cerbo, R., Masedu, F., De Caris, M., \& Sorge, G. (2010). Intensive intervention for children and adolescents with autism in a community setting in Italy: A single-group longitudinal study. Child and Adolescent Psychiatry and Mental Health, 4(1), 23-31.

Warren, Z., McPheeters, M. L., Sathe, N., Foss-Feig, J. H., Glasser, A., \& Veenstra-VanderWeele, J. (2011). A systematic review of early intensive intervention for autism spectrum disorders. Pediatrics, 127(5), 1303-1311.

Weiss, M. J. (1999). Differential rates of skill acquisition and outcomes of early intensive behavioral intervention for autism. Behavioral Interventions, 14(1), 3-22.

Willians, L. A., \& Aiello, A. L. R. (2001). Inventário Portage operacionalizado. São Paulo: Mennon. 